\title{
Finite-DifFERENCE TIME-DOMAIN SimUlation OF Light SCATTERING FROM Single CeLls
}

\author{
Andrew Dunn, Colin Smithpeter, Ashley J. Welch, and Rebecca Richards-Kortum \\ Biomedical Engineering Program, ENS 610, University of Texas, Austin, TX 78712 \\ (Paper JBO-098 received June 30, 1996; revised manuscript received Jan. 12, 1997; accepted for publication Apr. 4, 1997. )
}

\begin{abstract}
The finite-difference time-domain (FDTD) technique is used to compute light scattering from biological cells in two dimensions. Results are presented for the computed scattering patterns of cells containing multiple organelles. This method provides considerably more flexibility than Mie theory because of its ability to model inhomogeneous objects such as cells. (c) 1997 Society of Photo-Optical Instrumentation Engineers. [S1083-3668(97)00203-7]
\end{abstract}

Keywords light scattering, tissue optics, FDTD.

\section{INTRODUCTION}

Recently there has been considerable interest in the optical properties of tissue in the near-infrared region for imaging and diagnostic applications where scattering is dominant. Much of this has been stimulated by the success of confocal ${ }^{1}$ and optical coherence tomography (OCT) imaging ${ }^{2}$ of human tissue in vivo with high resolution. Relating the measured changes in tissue scattering to physiological differences on the cellular level, such as those between normal and cancerous tissue, has been difficult since the mechanisms by which cellular changes affect light scattering are not fully understood. This is primarily due to the difficulty in describing scattering at the cellular level in an inhomogeneous medium.

Scattering in tissue arises from local changes in the index of refraction between cell components and other small tissue structures, and the scale over which these changes occur varies from tissue to tissue. Traditionally these local variations in index are characterized by a bulk or macroscopic scattering coefficient $\mu_{s}$, which represents the probability of scattering per unit length. An understanding of the relationship between the bulk scattering properties of tissue and the local variations in the index of refraction on the cellular level will aid in the interpretation and development of optical diagnostic techniques.

In this paper we discuss the application of the FDTD technique for modeling light scattering from cells in two dimensions. This technique allows the computation of the electromagnetic fields within and around a cell with high accuracy for cells of arbitrary shape and with any number of compo-

Address all correspondence to Rebecca Richards-Kortum. Tel: (512) 4717827; fax: (512) 471-0616; e-mail: kortum@mail.utexas.edu nents. The effects of various organelles on the farfield scattering patterns of cells are examined with the FDTD method. The scattering pattern of cells and tissues is an important factor in determining the nature of light propagation in tissue and can influence the amplitude of backscattered light in elastic scattering measurements of tissue ${ }^{3}$ as well as the amount of fluorescence escaping the tissue surface. $^{4}$

\section{FDTD METHOD}

First proposed by $\mathrm{Yee}^{5}$ in 1966, the FDTD algorithm has been widely used in electromagnetic modeling applications. ${ }^{6-8}$ The FDTD method will be briefly outlined; a more detailed explanation can be found in Refs. 9 and 10. Using Yee's algorithm, Maxwell's curl equations are numerically solved by discretizing them in space and time. In two dimensions the discretization yields three explicit finite-difference equations for transverse magnetic (TM) and transverse electric (TE) polarized waves. Central difference approximations are used for the derivatives with the electric and magnetic fields temporally and spatially offset. The finite-difference equations for the $E_{z}, H_{x}$, and $H_{y}$ fields in a lossless medium, assuming a conductivity $\sigma$ of zero, for TM polarization are

$$
\begin{aligned}
E_{z}^{n+1}(i, j)= & E_{z}^{n}(i, j)+\frac{\delta t}{\epsilon(i, j) \Delta}\left[H_{y}^{n+1 / 2}(i+1 / 2, j)\right. \\
& -H_{y}^{n+1 / 2}(i-1 / 2, j)+H_{x}^{n+1 / 2}(i, j-1 / 2) \\
& \left.-H_{x}^{n+1 / 2}(i, j+1 / 2)\right]
\end{aligned}
$$




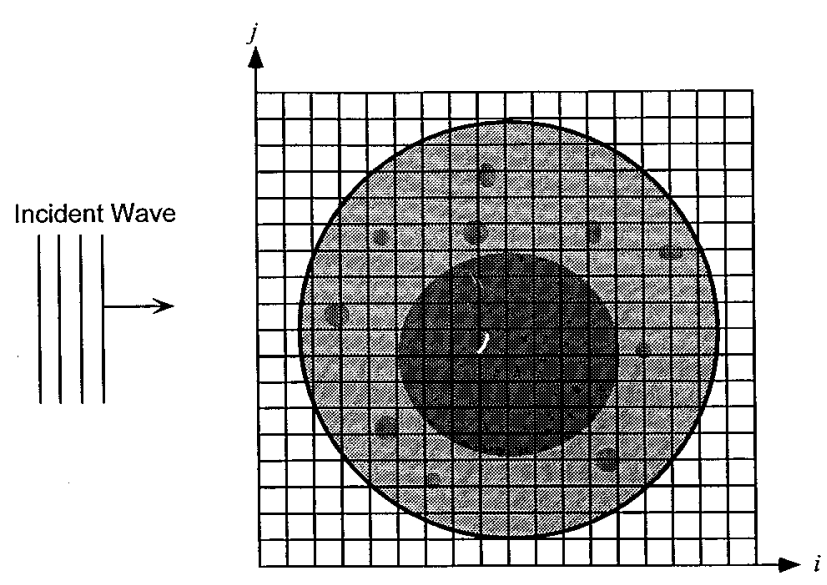

Fig. 1 Geometry for typical cell used in the FDTD simulation. The cell is constructed by assigning different refractive indices at each grid point.

$$
\begin{aligned}
H_{x}^{n+1 / 2}(i, j+1 / 2)= & H_{x}^{n-1 / 2}(i, j+1 / 2)+\frac{\delta t}{\mu \Delta}\left[E_{z}^{n}(i, j)\right. \\
& \left.-E_{z}^{n}(i, j+1)\right] \\
H_{y}^{n+1 / 2}(i+1 / 2, j)= & H_{y}^{n-1 / 2}(i+1 / 2, j) \\
& +\frac{\delta t}{\mu \Delta}\left[E_{z}^{n}(i+1, j)-E_{z}^{n}(i, j)\right],
\end{aligned}
$$

where $\delta t$ is the time step and $\Delta$ is the spacing between adjacent grid elements. In Eqs. (1) to (3), the spatial grid indices in the $x$ and $y$ directions are specified by $i$ and $j$, respectively, and the time index by $n$. The permittivity of the medium at point $(i, j)$ is indicated by $\epsilon(i, j)$ and the permeability $\mu$ is assumed to be constant.

The electric and magnetic fields are updated at all interior grid points at alternate half time steps using Eqs. (1) through (3). To prevent artificial reflections along the edges of the grid, a suitable boundary condition, such as the Mur ${ }^{11}$ or Liao absorbing boundary condition, ${ }^{12}$ must be applied. In this work the Liao boundary condition was used to simulate propagation in an unbounded medium.

The FDTD geometry is shown in two dimensions in Figure 1. At each grid point $(i, j)$, the dielectric constant for the medium is specified. By assigning different values to each of the organelles, the cell can be constructed as a dielectric object with any number of components. The portions of the cell are represented by different gray levels in Figure 1, and the index of refraction is related to the dielectric constant by $n=\sqrt{\epsilon_{r}}$. One source of error in FDTD calculations is the approximation of smooth surfaces as stair-stepped boundaries due to the Cartesian grid. ${ }^{13}$ To improve the stair casing approxima-
Table 1 Index of refraction values used in the FDTD simulations, taken from previously published sources.

\begin{tabular}{lcc}
\hline Cell component & Refractive index & Reference \\
\hline Surrounding medium & 1.35 & $\mathrm{a}$ \\
Cytoplasm & 1.37 & $\mathrm{~b}$ \\
Cell membrane & 1.46 & $\mathrm{c}$ \\
Nucleus & 1.39 & $\mathrm{~b}$ \\
Melanin & 1.7 & $\mathrm{~d}$ \\
\hline
\end{tabular}

a R. Barer and S. Joseph, "Refractometry of living cells," Quart. J. Microscop. Sci. 95, 399-423 (1954).

${ }^{b}$ A. Brunsting and P. Mullaney, "Differential light scattering from spherical mammalian cells," Biophys. J. 14, 439-453 (1974).

c J. Maier, S. Walker, S. Fantini, M. Franceschini, and E. Gratton, "Possible correlation between blood glucose concentration and the reduced scattering coefficient of tissues in the near infrared," Opt. Lett. 19, 2062-2064 (1994)

d I. Vitkin, J. Woolsey, B. Wilson, and R. Anderson, "Optical and thermal characterization of natural (sepia oficinalis) melanin," Photochem. Photobiol. 59, 455-462 (1994).

tion, the index of refraction at each point in the grid was taken to be the average value of all surrounding points.

A sinusoidal plane wave source of the form $\sin (\omega t)$ was applied at $x=0$ and the algorithm stepped in time until steady state was reached throughout the grid, which typically required three to four passes through the entire grid. After steady state is reached, the fields are known on the entire grid which lies in the near field. However, to compute the total scattering pattern in the far field, a transform from the near field to the far field is required. This involves weighting the tangential field components on a closed surface surrounding the scatterer with the free space Green's function, and integrating over the surface. The details of the transform can be found in Ref. 14 .

Due to stability considerations, the maximum grid spacing $\Delta$ is usually taken to be $\lambda / 10$ and the maximum time step $\delta t$, is limited to ${ }^{15}$

$$
\delta t=\frac{\Delta}{c \sqrt{2}},
$$

where $c$ is the speed of light in the medium. In this work the grid spacing was $\lambda / 60$, where $\lambda=900 \mathrm{~nm}$, to accommodate the small cellular structures, and the temporal step size was $\Delta / 2 c$.

\section{RESULTS}

The scattering patterns were computed for cells containing different organelles to determine the effect of each cell component on the total amount of scatter. The index of refraction values for each cell component used in the simulations are given in Table 1 and have been taken from previously pub- 


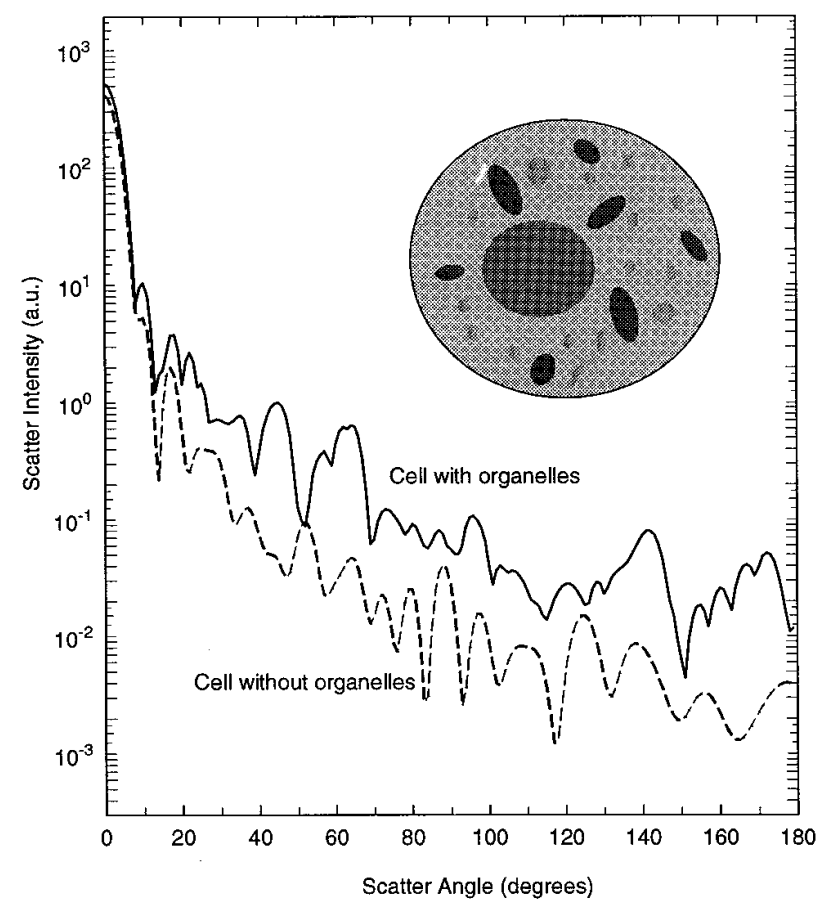

Fig. 2 Comparison of scattering patterns for cells with and without small organelles. The phase functions shown have not been normalized so that amplitude information has been preserved. The cell used in the simulations is shown in the inset with a cell diameter of $11 \mu \mathrm{m}$, a nucleus diameter of $3.5 \mu \mathrm{m}$, a membrane thickness of $15 \mathrm{~nm}$, and organelles ranging in size from 0.5 to $1.5 \mu \mathrm{m}$.

lished values. The scattering patterns presented in this paper have not been normalized so that amplitude information has been preserved.

The FDTD code was verified by comparison with the analytical solution for scattering from a two dimensional circular object, or infinite cylinder. ${ }^{16}$ The FDTD code agreed with the analytical solution to within $1 \%$ at all angles in the scattering pattern.

In Figure 2 the scattering functions of two cells with diameters of $11 \mu \mathrm{m}$ are plotted. In the first case the cell contains only a nucleus, membrane, and cytoplasm (dashed line); in the second case small organelles $(n=1.4)$ have been added to the cell (solid line) as shown in the inset. The scattering patterns are shown only for 0 to 180 deg since they are approximately symmetric from 180 to $360 \mathrm{deg}$. Each scattering pattern has been averaged over two perpendicular orientations of the cell and with the values from 180 to $360 \mathrm{deg}$ to smooth out the sharp peaks that arise from interference between the scattered waves. Although these individual peaks and valleys are predicted for a single cell, they will average out in multicell samples since all cells will have slightly different orientations.

It is important to understand the role of the nucleus in scattering because cancerous cells are characterized by a large nuclear-to-cytoplasmic ratio. ${ }^{17}$ Simulations to assess the effect of nucleus size on the scattering pattern are shown in Figure 3.

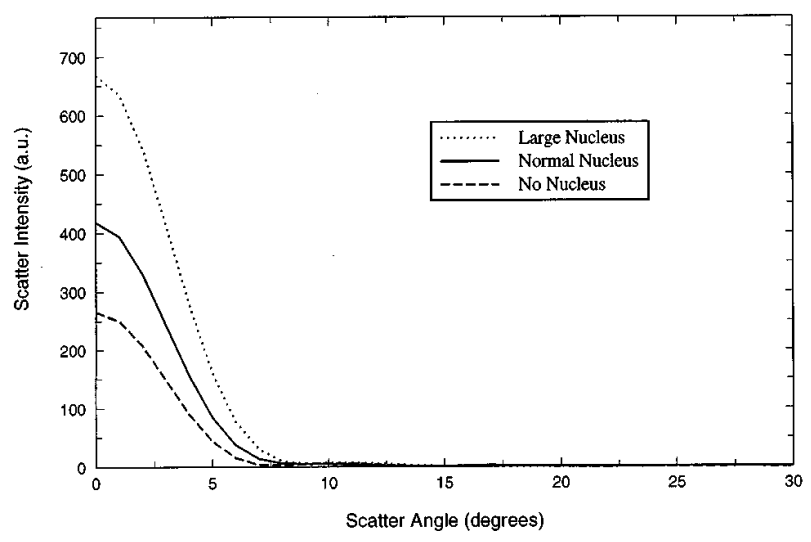

Fig. 3 Effect of the nucleus size on small-angle scatter. The plots shown are for cells containing a membrane, cytoplasm, and variable-sized nucleus. Inset: Full scattering pattern from 0 to 180 deg.

The scattering pattern is plotted for small angles since this is the region where the most significant differences occur. Cells containing large nuclei (diameter $\sim 6 \mu \mathrm{m}$ ) cause increased amounts of scattering at small angles relative to cells with normalsized nuclei (diameter $\sim 3 \mu \mathrm{m}$ ). In the absence of a nucleus, the amplitude of the scattering pattern decreases further.

The effect of melanin on the scattering pattern is demonstrated in Figure 4. The dashed curve is the scattering pattern from a cell containing only a nucleus, membrane, and cytoplasm, and the solid

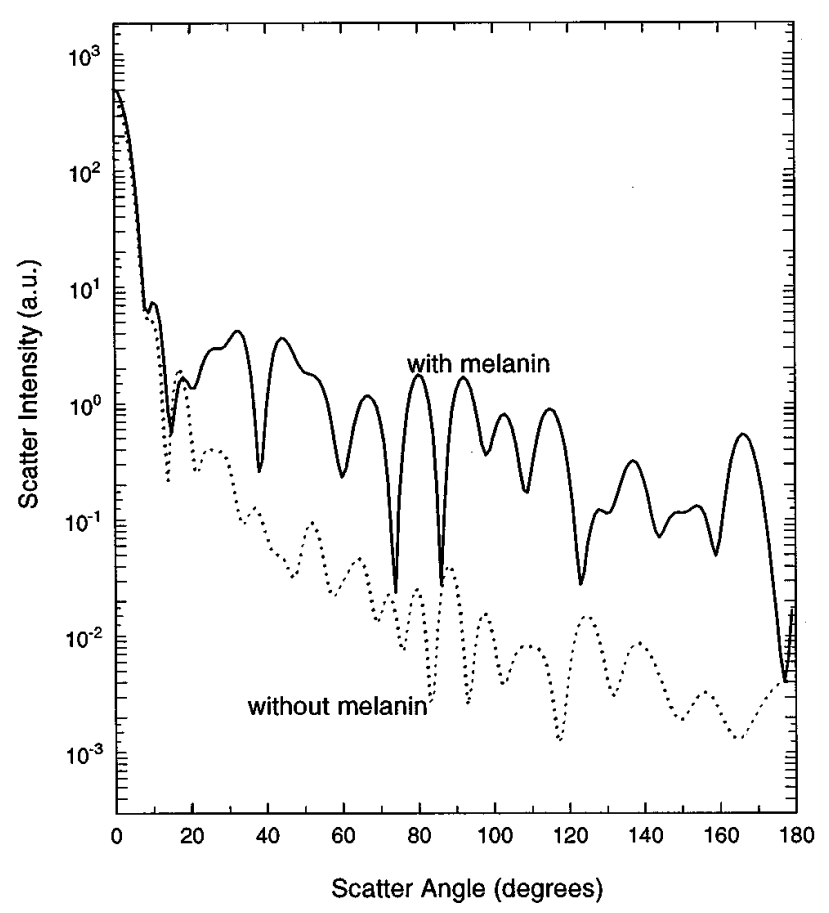

Fig. 4 Scattering patterns for a cell containing melanin granules (solid line) and a cell with no melanin (dotted line). The dimensions of the cell components are the same as those in Figure 2. 
line is the same cell with melanin granules added. The melanin granules had an average diameter of 1 $\mu \mathrm{m}$ and occupied approximately $15 \%$ of the cell area. The amount of scatter at high angles is up to two orders of magnitude greater than the same cell without melanin. The forward scatter component is slightly increased, but the primary difference lies at angles greater than about $30 \mathrm{deg}$. The absorption of melanin can be taken into account by using a complex index of refraction. For melanin, the imaginary part of the index $n^{\prime}$ was taken to be $0.004 .^{18}$

\section{DISCUSSION}

The scattering from a cell is highly peaked in the forward direction. Figure 2 demonstrates that small organelles can have a large effect on the scattering pattern. The total amount of scattered light, computed by integrating the pattern over all angles, was 1.7 times greater for the cell with small organelles than for the same cell without the organelles. This predicts that the small organelles can contribute significantly to the total scattering. This has been observed experimentally in rat liver cells, where mitochondria have been found to be the principal scatterer. ${ }^{19}$

The large nucleus causes a rise in the amount of forward scatter compared with the normal cell because its size is large compared with the wavelength. It does not play an important part in defining the shape of the scattering pattern other than at small angles. However, inhomogeneities within the nucleus would produce an increase in backscatter, as predicted in Ref. 20.

The role of melanin in tissue scattering has been largely overshadowed by its role as an absorber. Since melanin granules have an index of refraction significantly higher than other cell components ${ }^{18}$ and are small relative to visible and near infrared wavelengths, they act as strong isotropic scatterers, which is demonstrated in Figure 4. The large backscatter has been observed experimentally in in vivo confocal images of the skin, where the portions of the cells containing melanin look very bright relative to the rest of the cell. ${ }^{1}$ In tissue, the absorption properties of melanin will dominate due to the relatively long path length of a photon, but in single cells the increased scatter is dominant, particularly at near infrared wavelengths.

When a cell is modeled as a two-dimensional object, it is assumed that the cell extends infinitely in the third dimension so that a two-dimensional circular object is actually an infinite cylinder. Cells, however, are three-dimensional objects and it is difficult to quantitatively compare the scattering properties of two- and three-dimensional objects. However, two-dimensional results can be used to predict the relative effects of the presence of organelles on cell scattering. In Ref. 21, small organelles were found to play an important role in high- angle scattering from three-dimensional cells.
While the shapes of the scattering patterns of two and three-dimensional cells differ, the effects of organelles and spatial inhomogeneities in refractive index are similar. The advantage of using the twodimensional approximation is the significant decrease in computational requirements. Scattering from cells in two dimensions can be simulated on personal computers, while much larger supercomputers must be used for computations in three dimensions.

The FDTD technique has advantages over other single scattering techniques such as Mie theory, since it can model complex shapes and is not subject to any geometrical constraints. Previous work relying on Mie theory has approximated cells as concentric spheres ${ }^{17}$ and while this approximation is valid for certain types of cells, it cannot account for arbitrarily shaped cells containing organelles. The disadvantage of a model not subject to geometrical constraints such as FDTD or the coupled dipole method, ${ }^{22,23}$ is increased computational complexity. The coupled dipole method differs from the FDTD technique in that a particle is placed on a cubic grid and divided into subvolumes, each modeled as a dipole, and the electric field due to the incident field and the dipoles is computed. The grid spacing, and therefore the computational requirements, is limited by the wavelength to a maximum of approximately $\lambda / 10 .^{24}$ Because of its geometrical flexibility, the FDTD and coupled dipole methods could provide valuable insight into the angular distribution of scattered light from cells for flow cytometry applications ${ }^{25}$ that rely on differences in scattered light intensity from individual cells at one or more angles.

The main limitation of the FDTD method and the coupled dipole method at optical and near infrared wavelengths is that computer storage and computation times may become prohibitive for large geometries. While Monte Carlo techniques allow calculation of the light intensity distributions for large geometries, they do not provide any information about the electromagnetic fields in the tissue, which is important in OCT imaging.

\section{CONCLUSIONS}

We present the first application of the FDTD method for modeling light scattering from cells. The method is flexible enough to allow computation of scattering from cells of any shape and with any index of refraction profile. The twodimensional scattering pattern for cells with different combinations of organelles has been computed. The results show that small organelles play an important role in the scattering pattern and that melanin can have a significant effect on large-angle, single scattering events, and that the nuclear size influences the forward scatter behavior of the cell. 
Future work will focus on applying the technique to three dimensions and comparing the differences between two and three dimensions.

\section{Acknowledgment}

The authors would like to thank the High Performance Computing Facility at the University of Texas at Austin for a computer time grant on a Cray J90 supercomputer.

\section{REFERENCES}

1. M. Rajadhyaksha, M. Grossman, D. Esterwitz, R. Webb, and R. Anderson, "In vivo confocal scanning laser microscope of human skin: melanin provides strong contrast," J. Invest. Dermatol. 104, 946-952 (1995).

2. J. Izatt, M. Hee, D. Huang, A. Swanson, C. Lin, J. Schuman, C. Puliafito, and J. Fujimoto, "Micron-resolution biomedical imaging with optical coherence tomography," Opt. Photonics News 4, 14-19 (1993).

3. J. Mourant, J. Boyer, A. Hielscher, and I. Bigio, "Influence of the scattering phase function on light transport measurements in turbid media performed with small sourcedetector separations,"' Opt. Lett. 21, 546-548 (1996).

4. A. Welch, R. Richards-Kortum, E. Chan, G. Criswell, J. Pfefer, and S. Warren, "Propagation of fluorescent light," Lasers Surg. Med. (in press) (1997).

5. K. Yee, "Numerical solutions of initial boundary value problems involving Maxwell's equations in isotropic media," IEEE Trans. Antennas Propagation AP-14, 302-307 (1966).

6. D. Sullivan, D. Borup, and O. Gandhi, "Use of the finitedifference time-domain method in calculating EM absorption in human tissues," IEEE Trans. Biomed. Eng. 34, 148-157 (1987).

7. M. Piket-May and A. Taflove, "Electrodynamics of visiblelight interactions with the vertebrate retinal rod," Opt. Lett. 18(8), 568-570 (1993).

8. P. Yang and K. Liou, "Light scattering by hexagonal ice crystals: comparison of finite difference time domain and geometric optics models," J. Opt. Soc. Am. A 12, 162-176 (1995).

9. A. Taflove, Computational Electrodynamics: The FiniteDifference Time-Domain Method, Artech House, Norwood, MA (1995).

10. K. S. Kunz and R. J. Luebbers, The Finite Difference Time Domain Method for Electromagnetics, CRC Press, Boca Raton, FL (1993).
11. G. Mur, "Absorbing boundary conditions for the finitedifference approximation of the time-domain electromagnetic-field equations," IEEE Trans. Electromagnetic Compatibility EMC-23, 377-382 (1981).

12. Z. P. Liao, H. L. Wong, B. Yang, and Y. Yuan, "A transmitting boundary for transient wave analysis," Sci. Sin., Ser. A 27, 1063-1076 (1984).

13. H. S. Langdon and R. J. Luebbers, "Radiation and scattering from curved surface scatterers with stair-cased FDTD," in 10th Annual Review of Progress in Applied Computational Electromagnetics, Vol. 1, p. 467, Applied Computational Electromagnetics Society, Monterey, CA (1994).

14. R. Luebbers, D. Ryan, and J. Beggs, "A two-dimensional time-domain near-zone to far-zone transformation," IEEE Trans. on Antennas Propagation AP-40, 848-851 (1992).

15. A. Taflove and M. Brodwin, "Numerical solution of steadystate electromagnetic scattering problems using the timedependent Maxwell's equations," IEEE Trans. Microwave Theory Techn. MTT-23, 623-630 (1975).

16. H. V. de Hulst, Light Scattering by Small Particles, Dover Publications, New York (1981).

17. R. Meyer, "Light scattering from biological cells: dependence of backscatter radiation on membrane thickness and refractive index," Appl. Opt. 18, 585-588 (1979).

18. I. Vitkin, J. Woolsey, B. Wilson, and R. Anderson, "Optical and thermal characterization of natural (sepia oficinalis) melanin," Photochem. Photobiol. 59, 455-462 (1994).

19. B. Beauvoit, T. Kitai, H. Liu, and B. Chance, "Time-resolved spectroscopy of mitochondria, cells and rat tissues under normal and pathological conditions," in Proc. SPIE 2326, 127-136 (1995).

20. M. Kerker, D. Cooke, H. Chew, and P. McNulty, "Light scattering by structured spheres," J. Opt. Soc. Am. 68, 592601 (1978).

21. A. Dunn and R. Richards-Kortum, "Three-dimensional computation of light scattering from cells," IEEE J. Special Topics Quantum Electronics (in press) (1997).

22. A. Hoekstra and P. Sloot, "New computational technique to simulate light scattering from arbitrary particles," Particle Particle Systems Characterization 11, 189-193 (1994).

23. E. Purcell and C. Pennypacker, "Scattering and absorption of light by nonspherical dielectric grains," Astrophys. J. 186, 705-714 (1973).

24. A. Hoekstra and P. Sloot, "Dipolar unit size in coupled dipole calculations of scattering matrix elements," Opt. Lett. 18, 1211-1213 (1993).

25. H. M. Shapiro, Practical Flow Cytometry, Alan R. Liss, New York (1988). 